(n)

Noberef tuluer

Journal of Nonlinear Mathematical Physics

ISSN (Online): 1776-0852 ISSN (Print): 1402-9251

Journal Home Page: https://www.atlantis-press.com/journals/jnmp

\title{
On the dynamics of internal waves interacting with the equatorial undercurrent
}

Alan Compelli, Rossen Ivanov

To cite this article: Alan Compelli, Rossen Ivanov (2015) On the dynamics of internal waves interacting with the equatorial undercurrent, Journal of Nonlinear Mathematical Physics 22:4, 531-539, DOI: https://doi.org/10.1080/14029251.2015.1113052

To link to this article: https://doi.org/10.1080/14029251.2015.1113052

Published online: 04 January 2021 


\title{
On the dynamics of internal waves interacting with the equatorial undercurrent
}

\author{
Alan Compelli \\ School of Mathematical Sciences, \\ Dublin Institute of Technology, Kevin Street, \\ Dublin 8, Ireland \\ alan.compelli@mydit.ie \\ Rossen Ivanov* \\ Faculty of Mathematics, \\ Oskar-Morgenstern-Platz, 1, \\ University of Vienna, \\ 1090 Vienna, Austria \\ rossen.ivanov@univie.ac.at
}

Received 1 September 2015

Accepted 1 October 2015

\begin{abstract}
The interaction of the nonlinear internal waves with a nonuniform current with a specific form, characteristic for the equatorial undercurrent, is studied. The current has no vorticity in the layer, where the internal wave motion takes place. We show that the nonzero vorticity that might be occuring in other layers of the current does not affect the wave motion. The equations of motion are formulated as a Hamiltonian system.

Keywords: Internal waves, Equatorial undercurrent, shear flow, Hamiltonian system.
\end{abstract}

2000 Mathematics Subject Classification: 35Q35, 37K05, 74J30

\section{Introduction}

The dynamics of the Pacific Ocean, within a band of about $2^{\circ}$ latitude from the Equator, is characterised by a pronounced stratification, greater than anywhere else in the ocean; see [11]. A very sharp layer separates a shallow layer of relatively warm water (and so less dense) near the surface, from a deeper layer of denser, colder water. Since these distinct top and bottom layers differ by density and temperature, the sharp layer between them is termed pycnocline and sometimes thermocline. The difference in density across the thermocline is about $1 \%$. In addition, underlying non-uniform currents occur, see e.g. [13]. In a band approximately $300 \mathrm{~km}$ wide about the Equator, the underlying currents are highly depth-dependent: in a sub-surface layer, typically extending no more than about $100 \mathrm{~m}$ down, there is a westward drift that is driven by the prevailing trade winds; just below this there lies the Equatorial Undercurrent (EUC), an eastward jet whose core resides on the thermocline. Below the EUC, the motion dies out rapidly so that, at depths in excess of about $240 \mathrm{~m}$, there is an abyssal layer of essentially still water. Variety of waves in this region is observed, including long waves with wavelengths exceeding $100 \mathrm{~km}$ [11]. There is ample evidence of large-amplitude

*Permanent address: School of Mathematical Sciences, Dublin Institute of Technology, Kevin Street, Dublin 8, Ireland; email: rossen.ivanov@dit.ie 
internal waves, with relatively short wavelengths (typically a few hundreds of metres) and periods about 5-10 min; see [14].

We examine a simplified model of two-dimensional internal geophysical waves propagating along the Equator. Although the Coriolis force plays the role of a waveguide keeping the EUC and the occurring waves to propagate along the equator (and to remain essentially two dimensional) its impact on the dynamics of this two dimensional motion is rather small. Thus, for simplicity we neglect Coriolis forces.

The gravitationally induced internal waves propagate on the common interface (thermocline/pycnocline) between the upper and lower media of different densities. (We assume for simplicity that both media have constant densities.) In physical reality the ocean surface will have surface waves. We neglect the surface waves and hence consider the top surface to be a flat boundary like a rigid lid. For long and intermediate surface waves coupled with internal waves such an approximation is justified, cf. $[8,11]$. The boundary underneath is by an impermeable flat bed, which is a relevant approximation for the ocean bed, in the equatorial Pacific.

Recently, explicit nonlinear solutions for equatorially trapped waves were obtained in the Lagrangian framework, see $[4,12]$. Correspondingly, solutions for the internal waves have been described in [5]. However, both these types of solution are restricted to relatively short wavelengths, and they are realistic representations of the flow only for the region near the surface and in the neighbourhood of the thermocline, respectively.

By examining the governing equations of the system we provide a Hamiltonian formulation of the equations and with a variable transformation we show that it has a canonical Hamiltonian structure. The Hamiltonian formulation of surface waves over deep water has been noticed by Zakharov [15] and since then the Hamiltonian models have been used extensively for various approximations with linear and nonlinear PDEs, e.g. $[6,7,9,10]$.

As per Figure 1 we define the lower medium $\Omega_{1}$ as the domain $\left\{(x, y) \in \mathbb{R}^{2}:-h_{1}<y<\eta(x, t)\right\}$, the upper medium $\Omega_{2}$ as the domain $\left\{(x, y) \in \mathbb{R}^{2}: \eta(x, t)<y<h_{2}\right\}$ and the entire system $\Omega_{1,2}$ as the domain $\left\{(x, y) \in \mathbb{R}^{2}:-h_{1}<y<h_{2}\right\}$ where $\{y=\eta(x, t)\}$ describes the elevation of the common interface. The subscript $c$ will be used to denote evaluation at the common interface. The shear flow in a general form is

$$
U(y)= \begin{cases}-\sigma, & y=h_{2} \\ \kappa, & l_{2} \geq y \geq-l_{1} \\ 0, & y=-h_{1}\end{cases}
$$

and moreover $U(y)$ is a continuous function for $h_{2} \geq y \geq l_{2}$ and $-l_{1} \geq y \geq-h_{1}$. It is apparent that $h_{2}>l_{2}>0>-l_{1}>-h_{1}$.

The constants $\sigma, \kappa, l_{1,2}, h_{1,2}$ are positive (see Fig. 1). We use the subscript notation $i=\{1,2\}$ to represent the lower and upper media respectively. The next assumption is that the pycnocline is always in the strip $l_{2} \geq y \geq-l_{1}$, i.e.

$$
l_{2} \geq \eta(x, t) \geq-l_{1}
$$

for all $x, t$ so that all internal waves take place in this strip.

The velocities in the two media are decomposed into 'wave' and 'current' parts:

$$
\left\{\begin{array}{l}
u_{i}=\varphi_{i, x}+U_{i}(y)=\psi_{i, y} \\
v_{i}=\varphi_{i, y}=-\psi_{i, x}
\end{array}\right.
$$




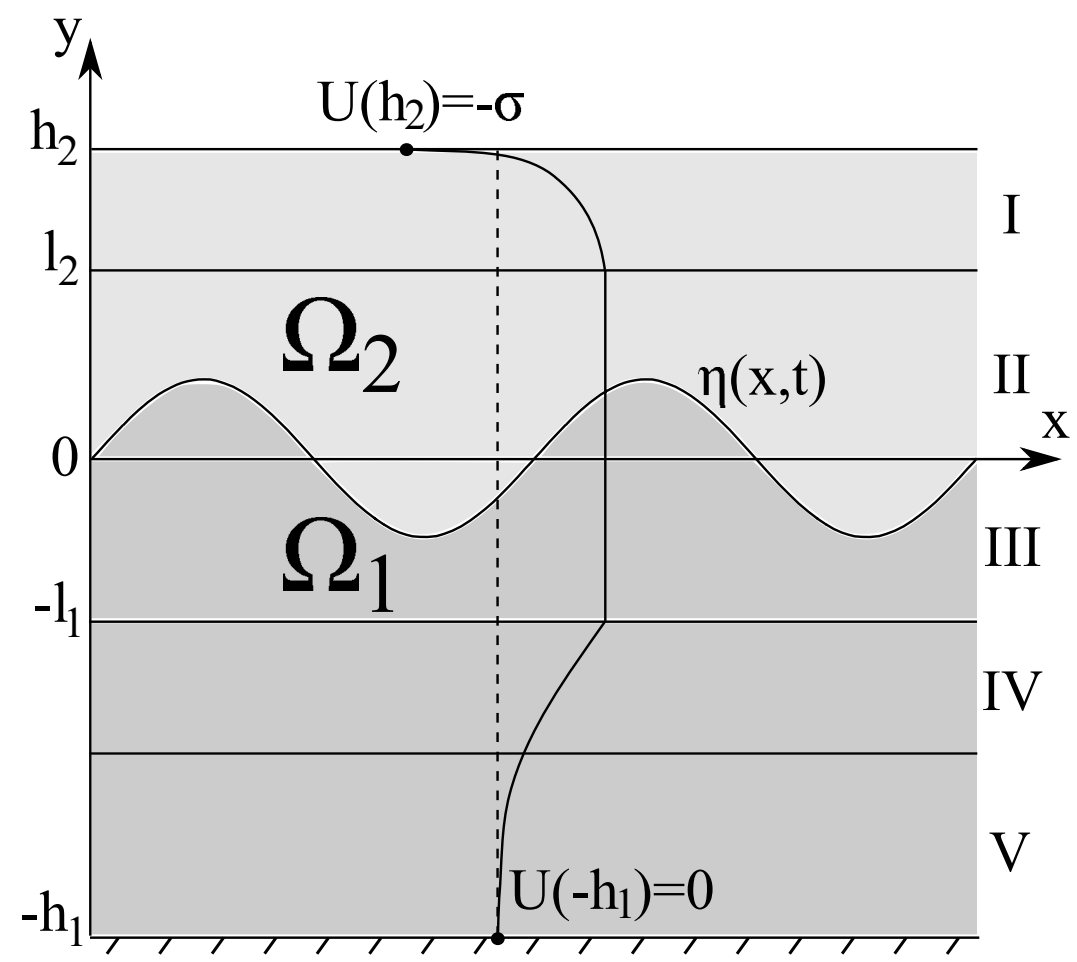

Figure 1. Setup: two domains $\Omega_{1}$ and $\Omega_{2}$ with densities $\rho_{1}$ and $\rho_{2}$. The shear profile $U(y)$ is provided qualitatively. The five layers I $-\mathrm{V}$ correspond to the description in [8]. The important feature is that the wave motion takes place in layers II and III where the vorticity is zero and $U(y)$ is constant. In the other layers $U(y)$ is continuous.

where without any ambiguity

$$
\begin{cases}U_{1}(y)=U(y), & h_{2} \geq y \geq \eta(x, t) \\ U_{2}(y)=U(y), & \eta(x, t) \geq y \geq-h_{1} .\end{cases}
$$

The non-lateral velocity flow, with propagation in the positive $x$-direction, is given by $\mathbf{V}_{i}(x, y, z)=\left(u_{i}, v_{i}, 0\right) \rho_{1}$ and $\rho_{2}$ are the respective constant densities of the lower and upper media and stability is given by the condition that

$$
\rho_{1}>\rho_{2}
$$

We assume that for large $|x|$ the amplitude of $\eta$ attenuates and hence make the following assumptions

$$
\lim _{|x| \rightarrow \infty} \eta(x, t)=0, \quad \lim _{|x| \rightarrow \infty} \varphi_{i}(x, y, t)=0 .
$$

\section{Governing Equations}

We write Euler's equation as:

$$
\mathbf{V}_{i, t}+\left(\mathbf{V}_{i} \cdot \nabla\right) \mathbf{V}_{i}=-\frac{1}{\rho_{i}} \nabla P_{i}+\mathbf{g}+\mathbf{F}_{c}
$$


where $P_{i}=\rho_{i} g y+p_{\mathrm{atm}}+p_{i}$ is the pressure at a depth $y, p_{\mathrm{atm}}$ is (constant) atmospheric pressure, $p_{i}$ is the dynamic pressure due to the wave motion, $g$ is the acceleration due to gravity (where $y$ points in the opposite direction to the center of gravity), $\mathbf{g}$ is the force due to gravity per unit mass, and the Coriolis force is neglected for simplicity. Its effect is not significant and also it is important to keep the waves one-dimensional.

Applying Equations (1.2) and noticing that in the strip $l_{2} \geq \eta(x, t) \geq-l_{1}$ we have $U(y)=\kappa=$ const. and velocities

$$
\left\{\begin{array}{l}
u_{i}=\varphi_{i, x}+\kappa=\psi_{i, y} \\
v_{i}=\varphi_{i, y}=-\psi_{i, x}
\end{array}\right.
$$

and thus there is no vorticity in the strip, i.e. $\Delta \psi(x, y)=0$. The equations can be written as

$$
\nabla\left(\varphi_{i, t}+\frac{1}{2}\left(\nabla \psi_{i}\right)^{2}\right)=\nabla\left(-g y-\frac{p_{i}}{\rho_{i}}\right)
$$

where $\nabla=\left(\partial_{x}, \partial_{y}\right)$. At the interface $p_{1}=p_{2}=p_{c}$ therefore we have the conservation law (Bernoulli condition) at the interface

$$
\rho_{1}\left(\left(\varphi_{1, t}\right)_{c}+\frac{1}{2}\left(\nabla \psi_{1}\right)_{c}^{2}+g \eta\right)=\rho_{2}\left(\left(\varphi_{2, t}\right)_{c}+\frac{1}{2}\left(\nabla \psi_{2}\right)_{c}^{2}+g \eta\right) .
$$

Writing the Bernoulli condition in terms of 'wave' potentials $\varphi_{i}$ only

$$
\left(\left(\rho_{1} \varphi_{1}-\rho_{2} \varphi_{2}\right)_{t}+\kappa\left(\rho_{1} \varphi_{1}-\rho_{2} \varphi_{2}\right)_{x}\right)_{c}+\rho_{1} \frac{1}{2}\left(\nabla \varphi_{1}\right)_{c}^{2}-\rho_{2} \frac{1}{2}\left(\nabla \varphi_{2}\right)_{c}^{2}+g \eta\left(\rho_{1}-\rho_{2}\right)=0 .
$$

The difference with the case without current is only the shift

$$
\partial_{t} \rightarrow \partial_{t}+\kappa \partial_{x}
$$

This also suggests the definition of $\xi_{i}:=\left(\varphi_{i}\right)_{c}=\varphi_{i}(x, \eta(x, t), t)$ as the interface velocity potential and hence the definition of ${ }^{\text {a }}$

$$
\xi:=\rho_{1} \xi_{1}-\rho_{2} \xi_{2}
$$

We will also use the following kinematic boundary conditions:

$$
\left\{\begin{array}{l}
\eta_{t}=-\eta_{x}\left(\left(\varphi_{i, x}\right)_{c}+\kappa\right)+\left(\varphi_{i, y}\right)_{c} \\
\left(\varphi_{1, y}\right)_{b}=\left(\varphi_{2, y}\right)_{l}=0
\end{array}\right.
$$

noting that $\mathbf{V}_{\mathbf{1}}\left(x,-h_{1}, 0\right)=\left(u_{1}, 0,0\right)$ and $\mathbf{V}_{\mathbf{2}}\left(x, h_{2}, 0\right)=\left(u_{2}, 0,0\right)$, where the subscripts $b$ and $l$ denote evaluation at the bottom (lower boundary) and lid (upper boundary) respectively. Again we observe only a shift $\partial_{t} \rightarrow \partial_{t}+\kappa \partial_{x}$ in comparison with the case $U(y) \equiv 0$ :

$$
\eta_{t}+\kappa \eta_{x}=-\left(\varphi_{i, x}\right)_{c} \eta_{x}+\left(\varphi_{i, y}\right)_{c}
$$

Therefore the time evolution of the variables, describing the dynamics of the internal waves $\xi, \eta$ does not depend on the shear outside the 'strip'; the constant flow in the strip leads only to a velocity shift with the velocity of the current $\kappa$ (in comparison to the currents free case).

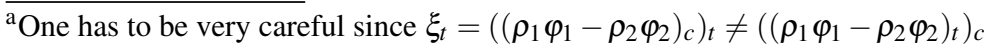




\section{Hamiltonian Formulation}

We will analyse the phenomenon from the Hamiltonian point of view. If we consider the system under study as an irrotational system the Hamiltonian, $H$, is given by the sum of the kinetic and potential energies as:

$$
H=\frac{1}{2} \int_{\mathbb{R}} \int_{-h_{1}}^{\eta} \rho_{1}\left(u_{1}^{2}+v_{1}^{2}\right) d y d x+\frac{1}{2} \int_{\mathbb{R}} \int_{\eta}^{h_{2}} \rho_{2}\left(u_{2}^{2}+v_{2}^{2}\right) d y d x+\frac{1}{2} \int_{\mathbb{R}}\left(\rho_{1}-\rho_{2}\right) g \eta^{2} d x .
$$

We try to express the Hamiltonian in terms of $\xi, \eta$. The kinetic energy of the lower layer is

$$
\begin{aligned}
K_{1} & =\frac{1}{2} \int_{\mathbb{R}} \int_{-h_{1}}^{\eta} \rho_{1}\left[\left(\varphi_{1, x}+U_{1}(y)\right)^{2}+\left(\varphi_{1, y}\right)^{2}\right] d y d x \\
& =\frac{\rho_{1}}{2} \int_{\mathbb{R}} \int_{-h_{1}}^{\eta}\left(\nabla \varphi_{1}\right)^{2} d y d x+\rho_{1} \int_{\mathbb{R}} \int_{-h_{1}}^{\eta}\left(\varphi_{1, x}\right) U_{1}(y) d y d x+\frac{\rho_{1}}{2} \int_{\mathbb{R}} \int_{-h_{1}}^{\eta} U_{1}^{2}(y) d y d x .
\end{aligned}
$$

We evaluate

$$
\begin{aligned}
& \int_{\mathbb{R}} \int_{-h_{1}}^{\eta}\left(\varphi_{1, x}\right) U_{1}(y) d y d x=\int_{\mathbb{R}}\left(\int_{-h_{1}}^{-l_{1}}+\int_{-l_{1}}^{\eta(x, t)}\right)\left(\varphi_{1, x}\right) U_{1}(y) d y d x \\
= & \int_{-h_{1}}^{-l_{1}}\left(\int_{\mathbb{R}}\left(\varphi_{1, x}(x, y)\right) d x\right) U_{1}(y) d y+\int_{\mathbb{R}} \int_{-l_{1}}^{\eta(x, t)}\left(\varphi_{1, x}\right) \kappa d y d x \\
= & \int_{-h_{1}}^{-l_{1}}\left(\int_{\mathbb{R}}\left(\varphi_{1, x}(x, y)\right) d x\right) U_{1}(y) d y+\kappa \int_{\mathbb{R}}\left(-\varphi_{1}(x, \eta) \eta_{x}+\partial_{x} \int_{-l_{1}}^{\eta(x, t)} \varphi_{1} d y\right) d x \\
= & -\kappa \int_{\mathbb{R}} \xi_{1} \eta_{x} d x
\end{aligned}
$$

since the integrals over total $x$ derivatives are zeroes due to the boundary conditions (1.5). The integral

$$
\begin{array}{r}
\int_{\mathbb{R}} \int_{-h_{1}}^{\eta} U_{1}^{2}(y) d y d x=\int_{\mathbb{R}}\left(\int_{-h_{1}}^{-l_{1}}+\int_{-l_{1}}^{\eta(x, t)}\right) U_{1}^{2}(y) d y d x \\
=\int_{\mathbb{R}}\left(\int_{-h_{1}}^{-l_{1}} U_{1}^{2}(y) d y\right) d x+\int_{\mathbb{R}}\left(\int_{-l_{1}}^{\eta(x, t)} \kappa^{2} d y\right) d x \\
=\int_{\mathbb{R}}\left(\int_{-h_{1}}^{-l_{1}} U_{1}^{2}(y) d y\right) d x+\kappa^{2} \int_{\mathbb{R}}\left(\eta(x, t)+l_{1}\right) d x
\end{array}
$$

gives an irrelevant constant, which can be absorbed by a proper renormalization and does not produce any dynamic terms, since the average elevation of the thermocline by definition is zero at $y=0$, i.e. $\int_{\mathbb{R}} \eta(x, t) d x=0$. The integrals over the upper layer produce

$$
\rho_{2} \kappa \int_{\mathbb{R}} \xi_{2} \eta_{x} d x
$$

and altogether the kinetic terms produce the extra term 


$$
\kappa \int_{\mathbb{R}}\left(\rho_{2} \xi_{2}-\rho_{1} \xi_{1}\right) \eta_{x} d x=-\kappa \int_{\mathbb{R}} \xi \eta_{x} d x
$$

This quantity itself is a conservation law, related to the energy of the overall translation of the system by a velocity $\kappa$, see [10]. The Hamiltonian of the system is given by:

$$
\begin{aligned}
H & =\frac{1}{2} \int_{\mathbb{R}} \int_{-h_{1}}^{\eta} \rho_{1}\left(\nabla \varphi_{1}\right)^{2} d y d x+\frac{1}{2} \int_{\mathbb{R}} \int_{\eta}^{h_{2}} \rho_{2}\left(\nabla \varphi_{2}\right)^{2} d y d x \\
& +\frac{1}{2} \int_{\mathbb{R}}\left(\rho_{1}-\rho_{2}\right) g \eta^{2} d x-\kappa \int_{\mathbb{R}} \xi \eta_{x} d x .
\end{aligned}
$$

We notice that the Hamiltonian does not depend on the $U(y)$ outside the strip. In order to express it in terms of the desired variables we introduce the Dirichlet-Neumann operators $G_{i}(\eta)$ given by

$$
G_{i}(\eta) \xi_{i}=\left(\varphi_{i, \mathbf{n}_{i}}\right) \sqrt{1+\left(\eta_{x}\right)^{2}}
$$

where $\varphi_{i, \mathbf{n}_{i}}$ is the normal derivative of the velocity potential $\varphi_{i}$, at the surface, for an outward normal $\mathbf{n}_{i}$, see more details in $[9,10]$. We define

$$
B:=\rho_{1} G_{2}(\eta)+\rho_{2} G_{1}(\eta) .
$$

We can express the Hamiltonian in terms of the conjugate variables $(\eta, \xi)$ as

$$
\begin{aligned}
H(\eta, \xi)=\frac{1}{2} \int_{\mathbb{R}} \xi\left(G_{1}(\eta) B^{-1} G_{2}(\eta)\right) \xi d x+\frac{1}{2} \int_{\mathbb{R}}\left(\rho_{1}-\rho_{2}\right) g \eta^{2} d x-\kappa \int_{\mathbb{R}} \xi \eta_{x} d x & \\
& =H_{0}(\eta, \xi)-\kappa \int_{\mathbb{R}} \xi \eta_{x} d x .
\end{aligned}
$$

where $H_{0}(\eta, \xi)$ is the Hamiltonian in absence of shear, $U(y) \equiv 0$ everywhere.

From (2.7), $\eta_{t}=\left(\psi_{i}(x, \eta, t)\right)_{x}$ and (2.5) can be written as a canonical Hamiltonian system

$$
\left\{\begin{array}{l}
\eta_{t}=\delta_{\xi} H \\
\xi_{t}=-\delta_{\eta} H
\end{array}\right.
$$

or

$$
\left\{\begin{array}{l}
\eta_{t}=\delta_{\xi} H_{0}-\kappa \eta_{x}, \\
\xi_{t}=-\delta_{\eta} H_{0}-\kappa \xi_{x} .
\end{array}\right.
$$

For the shifted variables $X=x-\kappa t$ and $T=t, \partial_{T}=\partial_{t}+\kappa \partial_{x}$

$$
\left\{\begin{array}{l}
\eta_{T}=\delta_{\xi} H_{0} \\
\xi_{T}=-\delta_{\eta} H_{0}
\end{array}\right.
$$

Therefore this system of equations has the usual canonical Hamiltonian form (same as the Hamiltonian form when there is no current) after the Galilean change of the coordinates, i.e. in a system travelling with velocity $\kappa$ together with the flow in the strip $l_{2} \geq y \geq-l_{1}$. 


\section{Linearization of the model equations}

By Taylor expanding the Dirichlet-Neumann operator we can represent it in terms of orders of $\eta$ as

$$
G_{i}(\eta)=\sum_{j=0}^{\infty} G_{i j}(\eta)
$$

with the constant, linear and quadratic terms given as

$$
\begin{aligned}
G_{i 0} & =D \tanh \left(h_{i} D\right) \\
G_{11}(\eta) & =D \eta D-G_{10} \eta G_{10} \\
G_{21}(\eta) & =-D \eta D+G_{20} \eta G_{20} \\
G_{i 2}(\eta) & =-\frac{1}{2}\left(D^{2} \eta^{2} G_{i 0}-2 G_{i 0} \eta G_{i 0} \eta G_{i 0}+G_{i 0} \eta^{2} D^{2}\right)
\end{aligned}
$$

where the operator $D$ is a Fourier multiplier equivalent to both the operation $-i \partial_{x}$ and the wavenumber $k$, i.e.

$$
D=-i \partial_{x}=k
$$

The operator $B$ can therefore be expressed as

$$
B=\rho_{1} \sum_{j=0}^{\infty} G_{2 j}(\eta)+\rho_{2} \sum_{j=0}^{\infty} G_{1 j}(\eta)
$$

and, also, the Hamiltonian can be represented as

$$
H(\eta, \xi)=\sum_{j=0}^{\infty} H^{(j)}(\eta, \xi)
$$

Using explicit expressions $H^{(2)}$ in terms of $\eta$ and $\xi$ is given by

$$
\begin{aligned}
H^{(2)}(\eta, \xi)=\frac{1}{2} \int_{\mathbb{R}} \xi \frac{D \tanh \left(h_{1} D\right) \tanh \left(h_{2} D\right)}{\rho_{1} \tanh \left(h_{2} D\right)+\rho_{2} \tanh \left(h_{1} D\right)} \xi d x & \\
& +\frac{1}{2} \int_{\mathbb{R}}\left(\rho_{1}-\rho_{2}\right) g \eta^{2} d x-\kappa \int_{\mathbb{R}} \xi \eta_{x} d x .
\end{aligned}
$$

Recalling (3.10) we calculate the linearized equations of motion as

$$
\begin{aligned}
\eta_{t}+\kappa \eta_{x} & =\frac{D \tanh \left(h_{1} D\right) \tanh \left(h_{2} D\right)}{\rho_{1} \tanh \left(h_{2} D\right)+\rho_{2} \tanh \left(h_{1} D\right)} \xi, \\
\xi_{t}+\kappa \xi_{x} & =-\left(\rho_{1}-\rho_{2}\right) g \eta .
\end{aligned}
$$

Looking for solutions in the form

$$
\left\{\begin{array}{l}
\eta(x, t)=\eta_{0} e^{i(k x-\Omega(k) t)} \\
\xi(x, t)=\xi_{0} e^{i(k x-\Omega(k) t)}
\end{array}\right.
$$

where, as usual $k$ is the wave number, $\Omega(k)$ is the angular frequency and $c(k)=\Omega / k$ is the wave speed, from the compatibility of the two equations we obtain the dispersion law 


$$
(c-\kappa)^{2}=\frac{g\left(\rho_{1}-\rho_{2}\right) \tanh \left(h_{1} k\right) \tanh \left(h_{2} k\right)}{k\left(\rho_{1} \tanh \left(h_{2} k\right)+\rho_{2} \tanh \left(h_{1} k\right)\right)} .
$$

In the case of infinite media as $h_{i} \rightarrow \infty$ then $\tanh \left(h_{i}\right) \rightarrow 1$ and (4.9) becomes

$$
(c-\kappa)^{2}=\frac{g}{k} \frac{\left(\rho_{1}-\rho_{2}\right)}{\left(\rho_{1}+\rho_{2}\right)} .
$$

The long-wave approximation of (4.9) is the case $k \rightarrow 0$ which gives

$$
(c-\kappa)^{2}=\frac{g h_{1} h_{2}\left(\rho_{1}-\rho_{2}\right)}{\rho_{1} h_{2}+\rho_{2} h_{1}} .
$$

Lastly, the consideration of the system as a single media system, i.e. $\rho_{2} \rightarrow 0$ in (4.9) gives

$$
(c-\kappa)^{2}=\frac{g}{k} \tanh \left(h_{1} k\right),
$$

which is the well known dispersion relation for the linear approximation of gravity water waves in a single medium irrotational system [3].

\section{Conclusions}

The shear flow in the layers, away from the thermocline (the strip where the internal waves occur) does not affect the dynamics of the internal waves. The results are complementary to the case where the vorticity is constant but with a finite jump at $y=\eta(x, t)$ considered in [1,2]. In the latter case vorticity does affect the wavespeed of the internal waves, cf. with [6].

\section{Acknowledgements}

A.C. is funded by the Fiosraigh Scholarship Programme of Dublin Institute of Technology. The support of the FWF Project I544-N13 "Lagrangian kinematics of water waves" of the Austrian Science Fund is gratefully acknowledged by R.I. The authors are grateful to Prof. A. Constantin for many valuable discussions and to an anonymous referee for several very useful suggestions.

\section{Bibliography}

[1] A. Compelli, Hamiltonian formulation of 2 bounded immiscible media with constant non-zero vorticities and a common interface, Wave Motion 54 (2015) 115-124.

[2] A. Compelli, Hamiltonian approach to the modeling of internal geophysical waves with vorticity, Monatsh. Math. (2014), published online, DOI:10.1007/s00605-014-0724-1; available from http://arrow.dit.ie/scschmatart/178/.

[3] A. Constantin, Nonlinear water waves with applications to wave-current interactions and tsunamis (SIAM, Philadelphia, 2011).

[4] Constantin, A., An exact solution for equatorially trapped waves, J. Geophys. Res.-Oceans 117 (2012) C05029.

[5] Constantin, A., Some nonlinear, equatorially trapped, nonhydrostatic internal geophysical waves, $J$. Phys. Oceanogr. 44 (2014) 781-789.

[6] A. Constantin and R. Ivanov, A Hamiltonian approach to wave-current interactions in two-layer fluids, Phys. Fluids 27 (2015) 086603. 
[7] A. Constantin, R. Ivanov, E. Prodanov, Nearly-Hamiltonian structure for water waves with constant vorticity, J. Math. Fluid Mech. 9 (2007) 1-14.

[8] A. Constantin and R.S. Johnson, The dynamics of waves interacting with the Equatorial Undercurrent Geophysical \& Astrophysical Fluid Dynamics 109 (2015) 311-358.

[9] W. Craig, P. Guyenne, C. Sulem, Coupling between internal and surface waves, Nat. Hazards 57 (2011) 617-642.

[10] W. Craig, P. Guyenne, H. Kalisch, Hamiltonian long wave expansions for free surfaces and interfaces, Comm. Pure Appl. Math. 24 (2005) 1587-1641.

[11] A. Fedorov, J. Brown, Equatorial waves, Encyclopedia of Ocean Sciences (2009) 3679-3695.

[12] D. Henry, An exact solution for equatorial geophysical water waves with an underlying current. Europ. J. Mech. B/Fluids 38 (2013) 18-21.

[13] J.P. McCreary, Modeling equatorial ocean circulation, Ann. Rev. Fluid Mech. 17 (1985) 359-409.

[14] J.N. Moum, J.D. Nash and W.D. Smyth, Narrowband oscillations in the upper equatorial ocean. Part I: Interpretation as shear instability. J. Phys. Oceanogr. 41 (2011) 397-411.

[15] V. Zakharov, Stability of periodic waves of finite amplitude on the surface of a deep fluid, Zh. Prikl. Mekh. Tekh. Fiz. 9 (1968) 86-94 (in Russian); J. Appl. Mech. Tech. Phys. 9 (1968) 190-194 (English translation). 